\title{
Research and Design of Wireless Projection System Based on WLAN\&3G
}

\author{
Ting-ting Wang, Li Zhang \\ Department of Information and Engineering Zhengzhou \\ Chenggong University of Finance and Economics \\ Gongyi, China \\ wttwjl@163.com
}

\author{
Fang Wang \\ Department of Electronic and Information Engineering \\ Zhengzhou Sias International University \\ Zhengzhou China \\ 26084927@qq.com
}

\begin{abstract}
For large volume of existing projection device, active power, portable difficult issues such as the design of the wireless projection system based on WLAN and 3G. This paper achieved wireless transmission of projected information, supporting $3 G$ wireless network connection, the formation of a wireless LAN, a wireless routing, multi-user access and sharing 3G resources. To facilitate debugging and expansion, the system has the versatility to design a USB interface module.
\end{abstract}

Keywords-WLAN; 3G; coupling technology; wireless projection

\section{INTRODUCTION}

With the rapid development of PDA and laptop popularization and development of personal communication devices, has greatly contributed to the miniaturization and diversification of personal communication devices. On the other hand, multimedia messaging, mobile conferencing and mobile office and other new businesses are appearing. The usefulness and portability of terminal equipment are increasing required. In order to allow customers to insight into technologies or products, some of technical supporters and product promoters need to share computer information to many people. This requires information on their own laptops projected to facilitate people to share at the same time [1].

Most existing projection device has a large volume, active power, difficult to carry, etc. Practicality and portability of projection device cannot meet the growing mobile office, mobile conference and other business needs. Some technical data and product information need to share with customers by the technical supporters and product promoters while they introduced technical information or product performance to others. Customers can have a better understanding of the product or technology from the viewing angle. With the development of wireless communication network, its transmission rate continuously improve, data transmission business are increasing. Wireless communication technology has been played a huge role on many applications. Because of wireless data communication without wires, fast layout, good scalability, convenience, they are something unmatched in data communication with the wired. So the research of wireless data transmission system has a very important practical significance. This paper describes a wireless projection system based on WLAN and 3G technology solutions.

\section{WLAN AND 3G NETWORK CONVERGENCE TECHNOLOGY}

\section{A. WLAN and $3 G$ respective characteristics}

WLAN (Wireless Local Area Networks), which is a short-range wireless transmission technology, but also a kind of wireless networking technology, based on IEEE802.11 standard. As a complement and extend the IEEE802.11 standard, as well as IEEE802.11a, IEEE802.11b, ..., IEEE802.11i etc [2]. In these protocols, the most common products are based on the protocol IEEE802.11b. WLAN radio can cover a part of area by deploying access point (AP). The most common access point is a wireless router. Then in effective range covered by the waves of wireless router can be connected to the Internet using WLAN connection. To be able to access the WLAN network place known as hot spots.

WLAN biggest advantage is the higher transmission speeds, up to $54 \mathrm{Mbps}$, The signal is weak or there is interference, bandwidth is adjustable to $5.5 \mathrm{Mbps}$, 2Mbps and $1 \mathrm{Mbps}$, The bandwidth of the automatic adjustment, effectively guarantee the stability and reliability of the network. WLAN'S effective distance also is very long, in the open area, communication distance of up to 305 meters, in the closed area, communication distance of 76 meters to 76 meters. WLAN is also compatible with the existing various 802.11 DSSS equipment, convenient and integrated with the existing wired Ethernet networks, network costs are lower.

3G (3rd-generation) refers to support high-speed data transmission of cellular mobile communication technology. $3 \mathrm{G}$ can well realize the wireless roaming in the global scope, and processing the images, music, video and other forms of media, including Webpage browsing, telephone conference, e-commerce and other information services. 3G provides data rates generally in more than hundreds of kbps.

The 3G standards are WCDMA (European Edition), CDMA2000 (American version) and TD-SCDMA (China version). TD-SCDMA is formulated by the Chinese alone, $3 \mathrm{G}$ standard, has the characteristics of low radiation, known as the green 3G. TD-SCDMA will intelligent wireless, synchronous CDMA, software radio to dissolve into the current international advanced technology, as well as the 
spectrum efficiency, support for business flexibility, the unique advantages of frequency agility and cost, etc. In addition, because of the huge market in mainland China. The standard is in major telecom equipment manufacturers, equipment factory are more than half of the world announced that can support TD - SCDMA standard.

\section{B. The advantage of WLAN and $3 G$ convergence technology}

WLAN data rate is high, but the wireless coverage is small and the support for mobility is poor, on the contrary, the support of $3 \mathrm{G}$ network for mobility is better, but the data transfer rate is low, two kinds of network technology in the mobile communication technology will achieve the fusion of local development, each will develop their advantage, foster strengths and circumvent weaknesses, complementary trend embodied in the following aspects [3, 4]:

(1) Voice and Vo-WLAN

Relative to meet big traffic, many users of $3 \mathrm{~g}$ technology, WLAN network based on IP technology is more suitable for business of broadcasting speech (PTT, multilateral meetings, long-distance calls, advertising, etc.).

(2) Wide area coverage and area coverage under the data services

Relative to the large coverage of $3 G$ technology, fast moving when still can keep the characteristics of the data rate of 144 kbps. WLAN technology in a specific area meeting the needs of users of high-speed data transmission has the absolute advantage.

(3) The use of wireless channel resources

Distribution of $3 G$ frequency resource is limited, and data services for channel utilization rate is extremely high, so it will affect the number of voice users that accesses at the same time. If a particular area planning (crowded areas such as business center) in the data business is transferred to WiFi/WiMAX public data channel, which will greatly improve the 3G wireless network resource utilization.

(4) Combination between hand-held terminals and Laptop/PDA

High data transmission rate, always on Line and low cost of Laptop/PDA can meet the demand of the large amount of information. More convenience to carry for business users and small 3G handset can meet the needs of individual users to quick news.

\section{WLAN and $3 G$ convergence schemes}

At present, in view of the fusion between WLAN and 3G network, every standard organizations and companies have proposed some different solutions [5, 6]: mobile IP, Internet, loose coupling and tight coupling and so on. According to the different close combined degree between WLAN and 3G network, all the solutions can be basically divided into two broad categories: tight coupling and loose coupling.

Tight coupling technology, in network integration, make WLAN is completely another part of the $3 G$ networks. Commonly WLAN wireless interface used as carrier of UMTS, and then put the WLAN as an access network directly connected to the $3 \mathrm{~g}$ core network. At this time for 3 $\mathrm{g}$ network protocol stack and interface is implemented in the
WLAN system. Access to the user through the simulation of the mobile network base station, and then the network through UNC protocol conversion, eventually making the core equipment of mobile network feel differences between different wireless access technologies.

In a tight coupling mode, people use the standardized interface of mobile communication. This approach emphasizes the mobility, business and application layer on the surface of the comprehensive fusion, which embodies the $3 \mathrm{~g}$ and WLAN fusion is the ultimate goal of business layer and application layer. Tight coupling of the two networks can be seen as a unified whole, without the third party directly to achieve interoperability, and has the advantages of small switch delay. This way of integration of the disadvantage is that the same operators need to maintain the $3 \mathrm{~g}$ network and WLAN network, at the same time, and mobile terminal configuration becomes very complex. Because need to implement the corresponding $3 \mathrm{~g}$ WLAN interface protocol stack, and requirements on the WLAN network to realize the $3 \mathrm{~g}$ certification authorization mechanism and so on, lead to the complexity of network operations and implementation.

To complete network gateway between WLAN and 3G, it needs the third network which combine WLAN and network, but not directly to 3G network, because of the high speed WLAN data does not pass the 3G core network, and the end user can still experience the seamless access. Loose coupling way on the structure, still keep separate network structure and protocol, the two sides can belong to different administrative domains, only by the transformation of the gateway entities to achieve mutual agreement, through the mobile IP technology to implement the WLAN and 3G switch position in the process of management and authentication and billing.

Loose coupling mode of WLAN network and 3G network connectivity provides great flexibility, because the user data into the $3 \mathrm{G}$ core network, the safety of the two networks can be used in a separate mechanism mobility management and QoS mechanism, loose coupling allows deployment of WLAN network and 3G network respectively, so as to reduce the engineering and maintenance difficulty, therefore, the current WLAN and 3G network looselycoupled integration way widely applied in the market, this article also uses the loose coupling way to incorporate WLAN and $3 G$ network.

\section{WiRELESS PROJECTION SyStem BASED ON WLAN\&3G}

\section{A. Wireless projection system framework}

Wireless projection system based on WLAN\&3G including 3G wireless module, $802.11 \mathrm{~b} / \mathrm{g} / \mathrm{n}$ wireless module, USB interface module, multimedia processor module, projection module, Power management module. As shown in figure 1. 


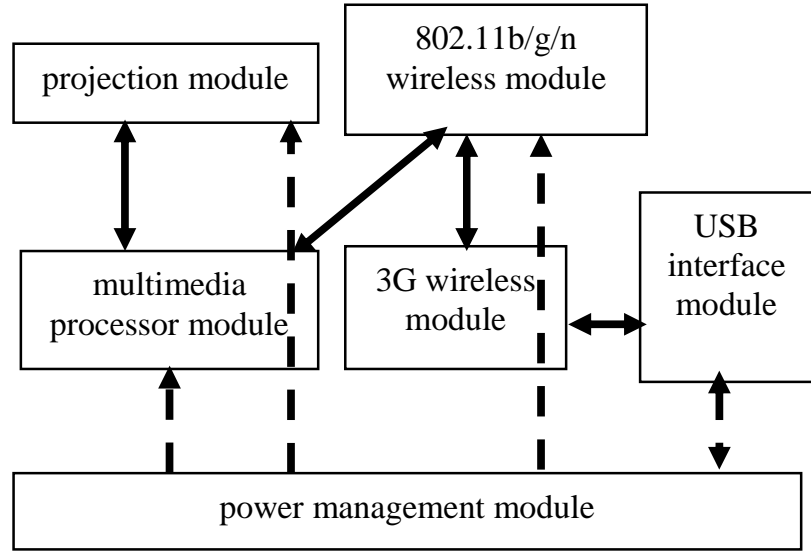

Figure 1 . The function module structure of wireless projection system

In the wireless projection system based on WLAN\&3G technology [7], 802.11.b/g/n wireless unit can complete the formation of wireless LAN, WIFI hot coverage and transmission of cloned or compressed computer screen image information to the multimedia processor. The information be sent to the projection unit after treatment. This mode realizes the wireless projection to meet mobile office, mobile phone, video conference, etc.

\section{B. Each module of wireless projection system based on WIFI\&3G technology function}

- The 3G wireless module is used to implement the 3G wireless network access, sets up $3 \mathrm{G}$ wireless data link, realizes the dynamic management of system resources. Users can through a wireless projection device based on WLAN / 3G, 3G wireless unit connected to the 3G network. They can experience of 3G wireless Internet, it can meet business requirements of user data such as multimedia messaging, online games, download music, improve the needs of the users' personal communication and entertainment.

- The user through a projection device based on wireless WIFI/3G technology, $802.11 \mathrm{~b} / \mathrm{g} / \mathrm{n}$ can form a wireless local area network (LAN), complete the WIFI coverage, realize the multi-user access, meet the demand of multi-user access to the Internet. At the same time, also can transmit cloning or compression of the computer screen image information to the multimedia processing unit, after processing, transmitted to the projection unit, realize the projection wireless.

- The USB interface module is mainly used for data transmission, the user interface of the parameters are passed to the device's 3G wireless unit, and realizes parameter configuration management system. The USB interface module can also provide battery charging interface and realize single user access $3 \mathrm{G}$ networks and users and devices, equipment and data transmission between devices, etc.
- Multimedia processor to achieve $802.11 \mathrm{~b} / \mathrm{g} / \mathrm{n}$ to the DVI interface protocol conversion, and will be for 802.11 $\mathrm{b} / \mathrm{g} / \mathrm{n}$ wireless transmit information of cloning or computer displaying image compression processing, into a DVI standard graphical information agreement, and transmitted to the projection unit, the laptop or handheld terminal screen image information reduction imaging, wireless projection function.

- The projection module mainly after receive disco pressed digital image information from multimedia processor reduction, and map the image information, then present to the user.

- Power management module implements on the battery, over-current protection, the power of the power supply and power distribution, and other functions, to achieve high performance and low power consumption.

When we start Wireless projection system, the working parameters of each interface module automatically by the system software configuration, or through a user interface configured manually, complete the data interaction of the interface unit and the automatic looking for landing, the 3G network.

\section{CONCLUSIONS}

Because the difficulty of (simple) circuit design is low, the system stability is strong, the cost is low, volume is more compact, easy to carry, 3G wireless access wireless, wireless local area network (LAN), projection, and other functions, not only meet the needs of users any time, any place. At the same time, the system uses the advanced projection technology, provide users with a mobile office, mobile Internet, mobile wireless local area network (LAN) work environment, in today's enterprise, business and other fields have broad market potential and prospect.

\section{REFERENCES}

[1] Xu Jie. Design and implementation of multimedia projector sharing system [D]. South-central nationalities university. 2012

[2] IEEE 802.11 work group. IEEE 802.11 Wireless Local Area Networks. http://grouper.ieee.org/groups/802/11/

[3] Kim Pyung-Soo, Kim Jung-Gon, Lee Jae-Kuk, et al. New authentication mechanism for vertical handovers between WLAN and $3 G$ network [C]. Proceedings of the Fifth IASTED International Conference on Communication Systems and Networks,2006,28(30): 26-31.

[4] Siddiqui F, Zeadally S, YaPrak E. Design architectures for 3G and IEEE802.11 WLAN integration [C]. 4th International Conference on Networking, 2005,3421:1047-1054.

[5] Phiri F A, Murthy M B R. WLAN-GPRS Tight Coupling Based Interworking Architecture with vertical handoff support [J]. Wireless Personal Communications, 2007,40(2):137-144.

[6] Lin Yida, Deng Shengwu. analysis of WLAN and 3G convergence [C]. 2012 The 2012 national wireless and mobile communication academic conference. 2012.09.01.

[7] Zhang Yaowei. A wireless projection based on WIFI and 3G technology [P]. China: 2011200448 